\title{
Viral Taxonomy
}

Marjorie J. Miller
From the Department of Pathology and Laboratory Medicine, UCLA Medical Center, Los Angeles, California

\begin{tabular}{|c|c|c|c|}
\hline Family & Subfamily & Genus & $\begin{array}{c}\text { Representative species pathogenic for } \\
\text { humans (no. of members) }\end{array}$ \\
\hline \multicolumn{4}{|l|}{ DNA Viruses } \\
\hline \multirow[t]{6}{*}{ Poxviridae } & Chordopoxvirinae & Orthopoxvirus & Vaccinia virus \\
\hline & & & Variola virus \\
\hline & & Parapoxvirus & Orf virus (contagious pustular dermatitis) \\
\hline & & & Pseudocowpox virus (milkers’ nodule) \\
\hline & & Molluscipoxvirus & Molluscum contagiosum virus \\
\hline & & Yatapoxvirus & Yaba monkey tumor virus, tanapox virus \\
\hline \multirow[t]{16}{*}{ Herpesviridae } & Alphaherpesvirinae & Simplexvirus & Human herpesvirus 1 \\
\hline & & & (Herpes simplex virus type 1 ) \\
\hline & & & Human herpesvirus 2 \\
\hline & & & (Herpes simplex virus type 2 ) \\
\hline & & & Herpes virus B \\
\hline & & & (Herpes simiae virus) \\
\hline & & Varicellovirus & Human herpesvirus 3 \\
\hline & & & (Varicella-zoster virus) \\
\hline & Betaherpesvirinae & Cytomegalovirus & Human herpesvirus 5 (cytomegalovirus) \\
\hline & & Roseolovirus & Human herpesvirus 6 \\
\hline & & & (human B lymphotropic virus) \\
\hline & & & Human herpesvirus 7 \\
\hline & Gammaherpesvirinae & Lymphocryptovirus & Human herpesvirus 4 \\
\hline & & & (Epstein-Barr virus) \\
\hline & & Rhadinovirus & Human herpesvirus 8 \\
\hline & & & (Kaposi's sarcoma-associated herpesvirus) \\
\hline Adenoviridae & & Mastadenovirus & Adenoviruses (49) \\
\hline \multirow[t]{3}{*}{ Papovaviridae } & & Papillomavirus & Papillomaviruses (77) \\
\hline & & Polyomavirus & $\mathrm{BK}$ virus (Polyomavirus hominis 1 ) \\
\hline & & & JC virus (Polyomavirus hominis 2) \\
\hline Parvoviridae & Parvovirinae & Erythrovirus & $\begin{array}{l}\text { B19 virus (human parvovirus, erythema } \\
\text { infectiosum or fifth disease) }\end{array}$ \\
\hline Hepadnaviridae & & Orthohepadnavirus & Hepatitis B virus \\
\hline \multicolumn{4}{|l|}{ RNA Viruses } \\
\hline \multirow[t]{4}{*}{ Reoviridae } & & Orthoreovirus & Reoviruses (3) \\
\hline & & Coltivirus & Colorado tick fever virus \\
\hline & & Orbivirus & Kemerovo virus \\
\hline & & Rotavirus & $\begin{array}{l}\text { Group } \mathrm{A}, \mathrm{B} \text {, and } \mathrm{C} \text { rotaviruses (numerous } \\
\text { serotypes) }\end{array}$ \\
\hline
\end{tabular}

Correspondence: Dr. Marjorie J. Miller, UCLA Clinical Laboratories, 11633

San Vicente Boulevard, 4th Floor, Los Angeles, California 90049.

Clinical Infectious Diseases 1997; 25:18-20

This article is in the public domain. 


\begin{tabular}{|c|c|c|c|}
\hline Family & Subfamily & Genus & $\begin{array}{c}\text { Representative species pathogenic for } \\
\text { humans (no. of members) }\end{array}$ \\
\hline \multicolumn{4}{|l|}{$\begin{array}{l}\text { RNA Viruses } \\
\text { (Continued) }\end{array}$} \\
\hline \multirow[t]{4}{*}{ Paramyxoviridae } & Paramyxovirinae & Paramyxovirus & Parainfluenza virus types 1 and 3 \\
\hline & & Morbillivirus & Measles virus \\
\hline & & Rubulavirus & $\begin{array}{l}\text { Mumps virus, parainfluenza virus types } 2,4 \mathrm{~A} \text {, } \\
\text { and 4B }\end{array}$ \\
\hline & Pneumovirinae & Pneumovirus & Respiratory syncytial virus \\
\hline \multirow[t]{2}{*}{ Rhabdoviridae } & & Vesiculovirus & Vesicular stomatitis virus \\
\hline & & Lyssavirus & Rabies virus \\
\hline Filoviridae & & Filovirus & Marburg and Ebola viruses \\
\hline \multirow[t]{3}{*}{ Orthomyxoviridae } & & Influenzavirus A & Influenza A virus \\
\hline & & Influenzavirus B & Influenza $B$ virus \\
\hline & & Influenzavirus $\mathrm{C}$ & Influenza $\mathrm{C}$ virus \\
\hline \multirow[t]{4}{*}{ Bunyaviridae } & & Bunyavirus & $\begin{array}{l}\text { Bunyamwera virus (Bunyamwera serogroup), } \\
\text { California encephalitis and LaCrosse } \\
\text { viruses (California serogroup), viruses of } \\
\text { other serogroups (mosquito-transmitted) } \\
\text { (>161) }\end{array}$ \\
\hline & & Hantavirus & $\begin{array}{l}\text { Hantaan virus (Korean hemorrhagic fever or } \\
\text { hemorrhagic fever with renal syndrome), } \\
\text { Muerto Canyon virus (Four Corners virus, } \\
\text { hantavirus pulmonary syndrome), and } \\
\text { others (rodent-associated) }(\sim 9)\end{array}$ \\
\hline & & Nairovirus & $\begin{array}{l}\text { Crimean-Congo hemorrhagic fever virus, } \\
\text { viruses of seven other serogroups (tick- } \\
\text { transmitted) }(>33)\end{array}$ \\
\hline & & Phlebovirus & $\begin{array}{l}\text { Sandfly fever viruses, Rift Valley fever virus } \\
\text { (sandfly-borne primarily) }(>30) \text {; } \\
\text { Uukuniemi and other viruses (tick- } \\
\text { transmitted) }(\sim 12)\end{array}$ \\
\hline \multirow[t]{4}{*}{ Arenaviridae } & & Arenavirus & Lymphocytic choriomeningitis virus \\
\hline & & & Lassa fever virus \\
\hline & & & Junin (Argentine hemorrhagic fever virus) \\
\hline & & & Machupo (Bolivian hemorrhagic fever virus) \\
\hline \multirow[t]{7}{*}{ Picornaviridae } & & Enterovirus & Polioviruses (3) \\
\hline & & & Coxsackie A viruses (23) \\
\hline & & & Coxsackie B viruses (6) \\
\hline & & & Echoviruses (30) \\
\hline & & & Enteroviruses $68-71$ (4) \\
\hline & & Rhinovirus & Common cold viruses $(>115)$ \\
\hline & & Hepatovirus & Hepatitis A virus (enterovirus 72 ) \\
\hline \multirow[t]{2}{*}{ Caliciviridae } & & Calicivirus & $\begin{array}{l}\text { Norwalk and Norwalk-like gastroenteritis } \\
\text { viruses (5) }\end{array}$ \\
\hline & & & $\begin{array}{l}\text { Hepatitis E virus (enterically transmitted } \\
\text { non-A, non-B hepatitis) }\end{array}$ \\
\hline Astroviridae & & Astrovirus & Human astroviruses $(7)$ \\
\hline Coronaviridae & & Coronavirus & Human coronaviruses $(2)$ \\
\hline
\end{tabular}




\section{Family}

Subfamily
Representative species pathogenic for humans (no. of members)

\section{RNA Viruses}

(Continued)

Flaviviridae
Flavivirus

Hepacivirus

Togaviridae

Retroviridae

The Subviral Agents

(Satellites,

Viroids, and

Agents of

Spongiform

Encephalopathies)

Satellites

Prions
Alphavirus

Rubivirus

HTLV-BLV viruses

Lentivirus

Deltavirus
St. Louis, Japanese B, and Murray Valley encephalitis viruses; yellow fever, dengue, and West Nile fever viruses (group B arboviruses, mosquito-borne) $(\sim 26)$

Kyasanur Forest disease, Omsk hemorrhagic fever, European and Far Eastern tick-borne encephalitis viruses (tick-borne) $(\sim 15)$

Vector-unassociated viruses $(\sim 17)$

Hepatitis $C$ virus (parenterally transmitted non-A, non-B hepatitis)

Hepatitis $G$ virus

Western, Eastern, and Venezuelan equine encephalitis viruses; Ross River, Sindbis, and Semliki Forest viruses (group A arboviruses, mosquito-borne) (27)

Rubella virus

Human T-cell lymphotropic viruses 1 and 2

Human immunodeficiency viruses 1 and 2

Hepatitis delta (D) virus

Kuru, Creutzfeldt-Jakob disease (CJD), Gerstmann-Straussler-Scheinker syndrome (GSS), fatal familial insomnia (FFI) 